\section{Psychische Störungen bei türkischen Migranten Inanspruchnahme einer Spezialambulanz}

\author{
Eva Kleinemeier ${ }^{1}$, Oktay Yagdiran ${ }^{1}$, Bilge Censi², Christian Haasen ${ }^{1}$ \\ ${ }^{1}$ Klinik für Psychiatrie \& Psychotherapie, Universitäts-Klinikum Hamburg-Eppendorf \\ 2 Klinikum Nord Ochsenzoll, Hamburg
}

psychoneuro 2004; 30 (11): 628-632

Die Inanspruchnahme psychiatrischer Einrichtungen durch Migranten mit psychischen Störungen ist deutlich reduziert. Gründe hierfür liegen in der erhöhten Schwelle von Seiten der Einrichtungen und am niedrigen Informationsstand über das Gesundheitssystem auf Seiten der Patienten. Die erhöhte Schwelle bei den Einrichtungen wird auf eine mangelnde kulturelle Kompetenz zurückgeführt. Diese Studie bewertet ein ambulantes Behandlungsangebot für türkisch sprechende Migranten. In den ersten sechs Monaten wurden 49 Patienten an die Ambulanz überwiesen. Die Hauptsymptome waren Depressivität, Somatisierung und Angst. 55\% erhielten die Diagnose einer neurotischen Störung (F4) und 24\% einer affektive Störung. Bei 15\% entsprach die Diagnose nicht der Überweisungsdiagnose, jedoch bestand kein Zusammenhang mit Sprachproblemen. Eine Spezialeinrichtung mit einem Psychiater, der die Muttersprache der Patienten spricht und die kulturellen Normen kennt, senkt die Schwelle der Inanspruchnahme.

n Deutschland leben fast zwei Millionen Menschen mit türkischer Staatsangehörigkeit, dies entspricht einem Anteil von 27,4\% aller nichtdeutschen Menschen (1). Die Mehrheit gehört zu den Arbeitsmigranten und deren Familien, die insbesondere in den 60er und 70er-Jahren angeworben wurden. Vor der Ankunft in Deutschland mussten sich alle Arbeitsmigranten einer Untersuchung des Gesundheitszustandes unterziehen, sodass der Anteil der kranken Migranten zum Zeitpunkt der Migration sehr gering war. Von keiner Seite wurde aber über mögliche psychische Folgen der Migration nachgedacht. Nach dem Stress-Modell der Migration von Slutzki (15) kommt es bei Migranten nicht unmittelbar während oder nach der Migration zu Belastungserscheinungen. Oft entwickeln Migranten erst nach einer
Phase der Euphorie - Slutzki spricht in diesem Fall von Überkompensation - psychische und körperliche Krankheitssymptome (15). Die Entscheidung für eine Migration kann sehr unterschiedliche Gründe haben. I.d.R. handelt es sich um eine belastende Entscheidung. Durch die Migration werden Menschen einer Vielzahl an Stressoren ausgesetzt: bedrohliche Lebensumstände, fragliche Zukunftsorientierung, Identitätskrisen, Entwurzelung, Trennungen etc. (2).

Es zeigt sich ein Defizit im psychosozialen Bereich sowohl in der Forschung als auch in den fehlenden Anstrengungen seitens der Psychiatrie, Migranten als besondere Patientengruppe zu berücksichtigen und ihnen spezielle Behandlungsangebote zu machen (2). Untersuchungen zu einer adäquaten Versorgung von Migranten sind selten. Es be- steht also die Notwendigkeit eines besseren Verständnis in der Beratung und Behandlung von psychiatrisch erkrankten Migranten, um eine optimale psychiatrische Versorgung zu gewährleisten und die Compliance der Patienten zu erhöhen. Nach Lazaridis (13) nehmen Migranten im Vergleich zu der einheimischen Bevölkerung die psychosozialen Versorgungsdienste des Gastlandes weit weniger in Anspruch. Diese Tatsache führt Lazaridis auf die organischen Krankheitsentstehungs-, Krankheitserklärungs- und Krankheitsdarbietungskonzepte zurück. Weiter beschreibt er übermäßige Somatisierungstendenzen bei vielen psychisch erkrankten Migranten.

Gründe für die geringe Inanspruchnahme liegen u.a. in den sprachlichen und kulturellen Barrieren und in der Orientierung des Hilfesystems an den Bedürfnissen westlicher Populationen, sowie in den negativen Konsequenzen, die eine psychiatrische Hospitalisierung mit sich bringen könnte (13). Wenn das Individuum keine andere Möglichkeit mehr sieht, seine Konflikte, sei es in der Familie, in seinem Umfeld oder am Arbeitsplatz, auszudrücken und auszutragen, flüchtet es in die ihm letzte Möglichkeit der Konfliktverarbeitung, die Krankheit. Dabei können nach Kiesel et al. (11) 
die Erkrankungen vielerlei Gestalt annehmen: sie können stärker psychisch oder überwiegend körperlich, also psychosomatisch sein. Das Krankheitserleben, Krankheitsempfinden, das Verhalten im Kranksein und das Krankheitsäußern sind dabei von bestimmten historischen, kulturellen und religiösen Hintergründen abhängig.

So fanden Günay \& Haag (7) in einer Studie in Allgemeinpraxen, dass türkische Frauen ihren Allgemeinarzt überwiegend mit körperlichen Symptomen und Antriebslosigkeit aufsuchen und somit eine höhere Prävalenz psychosomatischer und depressiver Störungen bei Migranten zu verzeichnen ist. Generell ist davon auszugehen, dass ein großer Teil der türkischen Patienten mit psychischen Symptomen sich in ihrer Heimat oder in Deutschland von traditionellen Heilern (Hodscha) behandeln lassen (6). Diese Hodschas sprechen die Sprache der Patienten, teilen i.d.R. eine ähnliche kulturspezifische Symptomwahrnehmung und -bewertung und nehmen eine aktive therapeutische Haltung an. Und genau dieses aktive Verhalten erwarten türkische Patienten von dem in ihrem Kulturkreis hoch angesehenen Arzt, während deutsche Ärzte von ihren Patienten eine differenzierte Eigenbeschreibung der Probleme und Symptome erhoffen (4).

Haasen et al. (8) konnten zeigen, dass in einem psychiatrischen Krankenhaus mit allgemeinem Versorgungsauftrag nur 6\% der Patienten Migranten waren, obwohl 12\% Migranten im Einzugsgebiet lebten, und dass im Gegensatz zu den übrigen Patienten (Deutsche 27\%) fast 50\% der Migranten die Diagnose einer schizophrenen Störung bekamen. Dies ließe sich darin begründen, dass die Schwelle zur Inanspruchnahme der Behandlung in der psychiatrischen Klinik u.a. aufgrund von fehlenden speziellen Angeboten zu hoch sei und daher nur die schweren psychiatrischen Störungen zur Aufnahme kämen.

Diese Studie stellt einen Versuch dar, die aktuelle Versorgung von türkischen Migranten im ambulanten Bereich zu untersuchen, da sich die neueren Studien zu den psychischen Störungen bei Migranten ausschließlich auf den stationären Bereich beziehen (u.a. 8, 10). Ausgangslage für diese Untersuchung war eine Studie von Haasen et al. (9), die die Versorgung türkischer Migranten in Allgemeinarztpraxen untersuchten.

\section{Methodik}

Um zu überprüfen, ob die Inanspruchnahme psychiatrischer Einrichtungen von türkisch sprechenden Menschen mit psychischen Beschwerden erhöht werden kann, wurde ein spezielles ambulantes Behandlungsangebot für türkische Migranten konzipiert. Verschiedene Hypothesen sollten überprüft werden:

- Ein spezielles ambulantes Behandlungsangebot für türkisch sprechende Migranten senkt die Schwelle für die Inanspruchnahme von psychiatrischen Einrichtungen

- Ein Behandlungsangebot im ambulanten Bereich wird vor allem von Patienten mit Depressionen und somatoformen Störungen in Anspruch genommen

- Durch mangelnde Kulturkenntnisse seitens der behandelnden Ärzte und durch die vorherrschenden Sprachprobleme werden psychische Erkrankungen bei Migranten verkannt und/ oder fehldiagnostiziert, was mit einer Spezialambulanz aufgefangen werden kann.

Alle Psychiater und Nervenärzte sowie türkischsprechende Ärzte in niedergelassenen Praxen in Hamburg wurden angeschrieben und über die Möglichkeit informiert, türkischsprechende Patienten mit psychischen Problemen in einer Spezialambulanz im Universitätsklinikum Eppendorf zur weiteren kulturspezifischen Diagnostik vorzustellen. Nach einer ausführlichen Untersuchung und Diagnosestellung durch einen Psychiater türkischer Herkunft wurden die Patienten von dem gleichen Arzt mit einem halbstandardisierten Interview befragt. In diesem Interview wurden soziodemographische Daten der Patienten erfasst sowie deren Migrations- und Krankheitsgeschichte. Die im Rahmen der Spezialsprechstunde gestellten Diagnosen wurden von dem verantwortlichen Arzt nach ICD-10 (3) codiert.

Der Erhebungszeitraum betrug sechs Monate (Januar bis Juni 1998). Einschlusskriterium war die türkische Herkunft der Patienten, wobei es keine Rolle spielte, ob sie in der Türkei oder in Deutschland geboren waren. Die Daten wurden mit Hilfe statistischer Verfahren $\left(\boldsymbol{x}^{2}, \mathrm{t}\right.$-Test, ANOVA) ausgewertet.

\section{Ergebnisse}

Zwischen Januar und Juni 1998 wurden insgesamt 49 Migranten vorgestellt. 30 Patienten wurden von einem niedergelassenen deutschen Arzt in die Spezialsprechstunde überwiesen, 17 Patienten von einem Arzt türkischer Herkunft und zwei Patienten von anderen niedergelassenen Ärzten. Im Durchschnitt besuchte jeder der 49 Patienten 1,4 mal $(S D=0,34 ; \operatorname{Min}=1$, Max = 5) die Spezialambulanz in der Psychiatrischen Klinik des Universitätsklinikums Eppendorf.

Von diesen 49 Migranten waren 25 weiblich (51\%) und 24 männlich (49\%). Das Durchschnittsalter lag bei $37,7$ Jahren ( $S D=10,5)$, wobei die Frauen im Durchschnitt 39 Jahre und die Männer 36 Jahre alt waren.

\section{- Soziodemographische Daten}

37 Patienten waren verheiratet, sechs Patienten waren ledig, vier getrennt lebend und jeweils ein $\mathrm{Pa}$ tient gab an, verwitwet zu sein bzw. in einer Beziehung zu leben. Dabei wurden keine geschlechtsspezifischen Unterschiede beobachtet. Im Durchschnitt betrug das Alter bei der Migration nach Deutschland 21,4 Jahre $(S D=8)$. Bei der Vorstellung in der Spezialambulanz lebten die Patienten durchschnittlich 17,3 Jahre in Deutschland (SD = 8,2). 82\% der Patienten haben Kinder, im Durchschnitt 2,3 Kinder (SD = 1).

In Bezug auf die berufliche Situation zeigte sich, dass 37\% der Patienten zum Erhebungszeitpunkt voll beschäftigt und 33\% arbeitslos waren. Zehn Frauen (20\%) bezeichneten sich als Hausfrauen, drei Patienten (6\%) waren bereits berentet und 
zwei (4\%) Personen teilzeitbeschäftigt. Bezüglich der beruflichen Situation unterschieden sich Männer und Frauen signifikant voneinander $\left(\boldsymbol{\chi}^{2}=\right.$ 17,2; p < 0,01, Tab. 1). Männer waren häufiger arbeitslos, aber auch häufiger vollzeitbeschäftigt im Vergleich zu den Frauen. Bezüglich der beruflichen Situation der Patienten ist auffällig, dass 85\% aller Patienten in der Spezialambulanz keine Schul- bzw. Berufsausbildung hatten.

Migrationsspezifische Daten

38 der untersuchten Migranten kamen alleine nach Deutschland und nur drei Personen sind hier geboren. 59\% der Migranten besaßen eine unbefristete Aufenthaltsgenehmigung und 20\% hatten bereits die deutsche Staatsangehörigkeit, während fünf Personen sich in einem laufenden Asylverfahren befanden, zwei Patienten Asyl gewährt bekommen hatten und zwei Patienten jeweils eine Duldung sowie eine befristete Aufenthaltsgenehmigung hatten.

Hinsichtlich der Deutschkenntnisse und der Aufenthaltsdauer in Deutschland fand sich ein signifikanter Unterschied: Die 15 Patienten $(26 \%)$ mit eher guten Deutschkenntnissen lebten im Durchschnitt 21 Jahren in Deutschland, im Gegensatz zu 34 Patienten (69\%) mit eher schlechten Deutschkenntnissen, die im Durchschnitt seit 15 Jahren in Deutschland lebten $(\mathrm{t}=3,3 ; \mathrm{p}=$ $0,01)$.

\section{Diagnosen, Psychopatholo- gie und weitere Krankheits- merkmale}

Das Ersterkrankungsalter, welches als das Alter der ersten psychiatrischen Diagnose definiert wird, lag in der Spezialambulanz im Durchschnitt bei 33,1 Jahren (SD = 10,6). Bei der Vorstellung im Universitätsklinikum lebten die Miganten durchschnittlich seit 17 Jahren (SD = 8,2 ) in Deutschland und waren im Mittel bereits seit 4,5 Jahren (SD = 5,5) erkrankt.

Bei 27 Migranten (55\%) wurde die Diagnose einer F4-Störung nach ICD-10 (F4 = Neurotische-, Belastungs- und somatoforme Störungen) gestellt, während zwölf Migranten

Tab. 1 Geschlecht und Arbeitssituation

\begin{tabular}{lcclll} 
& arbeitslos & Vollzeit & Teilzeit & Hausfrau & Rente \\
\hline weiblich & 4 & 7 & 2 & 10 & 2 \\
männlich & 12 & 11 & 0 & 0 & 1 \\
Gesamt & 16 & 18 & 2 & 10 & 3 \\
& & & & & $\chi^{2}=17,2 ; p<0,01$
\end{tabular}

\section{Tab. 2 Verteilung der ICD-10-Diagnosen}

\begin{tabular}{lllrlll} 
Geschlecht & F1 & F2 & F3 & F4 & F6 & Gesamt \\
\hline Männer & 2 & 2 & 6 & 12 & 2 & 24 \\
\hline Frauen & 0 & 3 & 6 & 15 & 1 & 25 \\
Gesamt & 2 & 5 & 12 & 27 & 3 & 49
\end{tabular}

F1 = Suchtstörung, F2 = Schizophrenie und wahnhafte Störungen, F3 = Affektive Störung, F4 = neurotische oder Belastungs- und somatoforme Störungen, F6 = Persönlichkeits- oder Verhaltensstörung

\section{Tab. 3 Diagnosen der Patienten mit depressiven Symptomen und Somatisierung}

\begin{tabular}{lllllll} 
Depressivität & F1 & F2 & F3 & F4 & F6 & Gesamt \\
\hline ja & 0 & 3 & 12 & 26 & 2 & 43 \\
nein & 2 & 2 & 0 & 1 & 1 & 6 \\
\hline Somatisierung & & & & & & \\
\hline ja & 0 & 3 & 10 & 19 & 3 & 35 \\
\hline nein & 2 & 2 & 0 & 8 & 0 & 14 \\
\hline
\end{tabular}

(24\%) die Diagnose einer affektiven Störung (F3) erhielten. Bei fünf Migranten wurde eine Schizophrenie oder wahnhafte Störung diagnostiziert, während die restlichen Migranten die Diagnose einer Persönlichkeits- und Verhaltensstörung (F7) oder einer Störung durch psychotrope Substanzen (F1) erhielten. In Bezug auf die psychiatrischen Diagnosen lassen sich keine geschlechtsspezifischen Unterschiede erkennen (Tab. 2).

Der psychopathologische Befund ergab, dass 88\% der Migranten unter depressiven Symptomen und 71\% unter Somatisierungen litten. 57\% wiesen Angstsymptome auf und 22\% zeigten psychotische Symptome. Bezüglich der Somatisierung konnte gezeigt werden, dass die Migranten ohne Somatisierungen mit durchschnittlich 17 Jahren länger in Deutschland vor Beginn der Erkrankung leben als die Migranten mit Somatisierungserscheinungen mit 11,6 Jahren $(t=2 ; \mathrm{p}<0,05)$. Somatisierungen und depressive Symptome treten bei Migranten mit den unter- schiedlichsten Diagnosen auf und nicht nur bei denen mit einer affektiven oder neurotischen und Belastungsstörung (Tab. 3). In Bezug auf depressive Symptome und Somatisierung zeigte sich ein signifikanter Unterschied insofern als Patienten mit einer depressiven Symptomatik eher somatisierten als Patienten ohne Anzeichen von Depressivität $\left(x^{2}=10,3 ; p=0,01\right)$.

Nur bei sieben Migranten stellte der Psychiater türkischer Herkunft in der Spezialambulanz eine andere Diagnose als der überweisende niedergelassene Arzt. Es konnte auch kein Zusammenhang zwischen den Sprachkenntnissen der Migranten und dem Auftreten eines Diagnosewechsels festgestellt werden.

\section{Diskussion}

Trotz der methodischen Schwäche dieser Untersuchung, dass nur 49 Migranten in der Spezialsprechstunde für Patienten türkischer Herkunft erfasst werden konnten, ergeben sich einige interessante Aspekte für die zukünftige Behandlung und 
Forschung. Ein möglicher Grund für die geringe Inanspruchnahme der Spezialambulanz kann in der schweren Zugänglichkeit liegen, da sich nur Migranten mit einem Überweisungsschein des niedergelassenen Arztes anmelden konnten. Aber auch Ängste vor Stigmatisierung, Diskriminierung bis zu Ängsten vor der Ausweisung aus Deutschland erklären die geringe Inanspruchnahme psychiatrischer und psychologischer Angebote bei Menschen mit türkischem Hintergrund und führen dazu, dass häufig auch nicht dem Allgemeinarzt von psychischen Problemen berichtet wird.

Aufgrund der kleinen Stichprobengröße kann kein Anspruch auf eine repräsentative Untersuchung erhoben werden. Die Hypothese, dass eine Spezialambulanz für Menschen türkischer Herkunft die Schwelle der Inanspruchnahme deutlich senkt, kann nicht eindeutig bestätigt werden, da wenige Migranten dieses Angebot in Anspruch genommen haben. Es kann aber auch daran liegen, dass die niedergelassenen Ärzte nur ihre „Problemfälle“ in die Ambulanz überwiesen hatten. Die Patienten, die das Angebot in Anspruch genommen hatten, gaben an, davon profitiert zu haben.

Die Tatsache, dass zwischen dem Migrationsalter und dem Ersterkrankungsalter zwölf Jahre liegen, bestätigt das Phasenmodell der Migration nach Slutzki (15), dass auf die Phase der Euphorie unmittelbar nach der Migration eine Phase der Dekompensation folgt, in der heftige Krisen mit somatischen und/oder psychiatrischen Symptomen auftreten. Eine ungünstige psychosoziale Konstellation zeigt sich für den Betroffenen in der Verschlechterung des psychischen Befindens. Der Zeitpunkt der Ersterkrankung markiert dann die psychische Dekompensation einer über Jahre schleichenden Entwicklung, die als spezifisch für Migranten angesehen werden kann.

Betrachtet man die Störungen, mit denen die Migranten die Spezialambulanz aufgesucht haben, so fällt auf, das die Mehrheit der Patienten an affektiven sowie neurotischen Belastungs- und somatoformen Störungen litten. Es scheint sich zu bestätigen, dass Migranten eine stationäre Behandlung eher bei schwerwiegenden psychiatrischen Erkrankungen in Anspruch nehmen $(8,10)$, während sie bei depressiven und somatoformen Störungen ambulante Hilfe suchen, insbesondere bei ihrem niedergelassenen Arzt. Diese Tatsache erlaubt aber nicht die Aussage, dass sich psychisch kranke Migranten vermehrt in der Allgemeinarztpraxis behandeln lassen (9). Mögliche Ursachen für die geringe Offenheit Menschen türkischer Herkunft, über psychische Probleme zu reden, sind u.a. Ängste vor Diskriminierung und Stigmatisierung, aber auch Sprachprobleme führen oft zu Missverständnissen.

Die Auswertung der psychopathologischen Befunde ergab einen sehr hohen Anteil depressiver und somatoformer Symptome in der gesamten Stichprobe (Tab. 3). Wie bereits schon bei den Diagnosen deutlich wurde, lässt sich mit den Analysen der Psychopathologie die Hypothese bestätigen, dass Menschen mit depressiven und somatoformen Symptomen eher Hilfe im ambulanten Bereich suchen. Dieses Ergebnisse stehen in Einklang mit früheren Studien, die insbesondere die Somatisierungstendenzen bei Migranten im ambulanten Bereich belegen konnten (7). Mit der „Somatization Study“ von Goldberg \& Bridges (5) konnte gezeigt werden, dass Migranten, Aussiedler und Flüchtlinge häufig mit Somatisierungen auf Stress und Schwierigkeiten reagieren, da sie ihre Probleme selten psychologisch erklären und zu psychotherapeutischen Hilfeangeboten wenig Zugang haben.

In diesem Zusammenhang ist es wichtig, auf die Jahre in Deutschland bei Migranten mit Somatisierungen einzugehen. Die Migranten ohne somatoforme Symptome lebten im Durchschnitt 5,4 Jahre länger in Deutschland vor Beginn der Erkrankung. Eine Erklärung für diesen signifikanten Unterschied kann sein, dass Menschen, die eine kürzere Zeit hier leben, im Verständnis der Entstehung, Bedeutung und Behandlung von Krankheiten durch ihre jeweilige Kultur geprägt sind. Je länger die Menschen in Deutschland leben, um so mehr nehmen sie die Kultur und auch das kulturelle Bild von Krankheit und Gesundheit des Gastlandes an. Eine solche Annahme des Krankheitsverständnisses kann sich u.a. in dem Eingestehen von psychischen Problemen zeigen.

Betrachtet man die Anzahl der Diagnosewechsel, wird deutlich, dass nur bei wenigen Patienten eine neue Diagnose gestellt werde musste. Und diese Wechsel scheinen in keinem Zusammenhang mit den Sprachkenntnissen zu stehen. Dies kann einerseits an der großen Kompetenz der überweisenden Ärzte liegen, aber auch an der sehr geringen Stichprobengröße. Dieses Ergebnis bestätigt, dass es beim Thema Migration und psychische Krankheit oft zu einer Überbewertung der Sprachproblematik kommt (9). Deutlich wird aber, dass es bei älteren Migranten häufiger $\mathrm{zu}$ Schwierigkeiten im Diagnoseprozess kommt, was mit der stärkeren Ausprägung historischer, kultureller und religiöser Normen erklärt werden kann. Die Hypothese, dass mangelnde Kulturkenntnisse der behandelnden Ärzte und Sprachprobleme zu einer hohen Zahl an Fehldiagnosen führen, kann nicht bestätigt werden. Hier zeigt sich die Notwendigkeit, dass es weiterer Studien bedarf, die mir Aspekte als Kultur und Sprache als Hindernisse in der Diagnose und Behandlung von Migranten einbeziehen.

Für die Migranten in der Spezialambulanz war es hilfreich, ein Gespräch mit einem Psychiater in ihrer Muttersprache zu führen, der ihre kulturellen Normen und kulturspezifische Symptomgestaltung kennt und sie somit nicht mit den Patienten des Gastlandes vergleicht. Es ist wichtig und notwendig, kulturspezifische diagnostische Kriterien zu entwickeln, um sie dann bei Migranten anzuwenden. Insbesondere die Somatisierungserscheinungen als Zeichen von psychischen Störungen sollten in diesen Kriterien mitberücksichtigt werden.

\section{Access to special out-patient clinic}

Access to mental health care is significantly reduced for migrants. Reasons lie in an increased threshold on 
Serie Migranten / Impressum

the side of services as well as lack of information about services on the side of the patients. The increased threshold is assumed to be due to lack of cultural competence. This study evaluates an outpatient clinic for Turkish speaking migrants. In the first 6 months 49 patients were seen. Main symptoms were depression, somatization and anxiety. 55\% received a diagnosis of neurotic disorder ( $F 4$ ) and $24 \%$ of affective disorder. In $15 \%$ of the cases the diagnosis differed from the one given by the physician who had sent the patient. A special service with a psychiatrist who speaks the native language and knows the cultural norms reduces the threshold in the access to care.

\section{Key words}

migration - depression - somatization - out-patient care - cultural competence

\section{Literatur}

1. Beauftragte der Bundesregierung für Ausländerfragen. Berlin, Bericht, 2000

2. Collatz J. Auf dem Wege in das Jahrhundert der Migration: Auswirkungen der Migrationsbewegungen auf den Bedarf an psychosozialer und sozialpsychiatrischer Versorgung. In: Koch E, Özek M, Pfeiffer WM (Hrsg.). Psychologie und Psychopathologie der Migration: deutsch-türkische Perspektiven. Freiburg: Lambertus 1995: 31-45

3. Dilling H, Mombour W, Schmidt MH. Internationale Klassifikation psychischer Störungen. ICD-10 Kapitel V (F). Göttingen, Verlag Hans Huber, 1993

3. Ete E. Ethnomedizinische Aspekte der Interaktion mit türkischen Patienten. In: Koch E, Özek M, Pfeiffer WM (Hrsg.). Psychologie und Psychopathologie der Migration: deutsch-türkische Perspektiven. Freiburg, Lambertus, 1995 4. Goldberg DP, Bridges KW. Somatic presentations of psychiatric illness in primary care setting. Journal of Psychosomatic Research 1988; 32: 137-144

5. Grube M. Darstellung eines türkisch-deutschsprachigen Verbundmodells zwischen psychiatrischer Klinik und psychosozialer Beratungsstelle. In: Koch E, Özek M, Pfeiffer WM (Hrsg.). Psychologie und Psychopathologie der Migration: deutsch-türkische Perspektiven. Freiburg, Lambertus, 1995: 199-205

6. Günay E, Haag A. Krankheit in der Emigration - Eine Studie an türkischen Patientinnen in der Allgemeinpraxis aus psychosomatischer Sicht. Psychotherapie, Psychosomatik und Medizinische Psychologie 1990; 40: 115-119

7. Haasen C, Lambert M, Yagdiran O, Krausz M. Psychiatric disorders among migrants in Germany: prevalence in a psychiatric clinic and implications for services and research. European Psychiatry 1997; 12: 305-310

8. Haasen C, Boyali A, Yagdiran O, Krausz M. Prävalenz psychischer Störungen bei Migranten in einer Allgemeinarztpraxis. Zeitschrift für Allgemeinmedizin 2000; 76: 512-516

9. Holzmann TH, Volk S, Georgi K, Pflug B. Ausländische Patienten in stationärer Behandlung in einer psychiatrischen Universitätsklinik mit Versorgungsauftrag. Psychiatrische Praxis 1994; 21: 106-108

10. Kiesel D. Kriechhammer-Yagmur S, von Lüpke H. (Hrsg.). Kränkung und Krankheit: Psychische und psychosomatische Folgen der Migration. Frankfurt/Main, Haag und Herchen, 1994

11. Lazaridis K. Psychiatrische Erkrankungen bei Ausländern - Hospitalisations- und nationalspezifische Inzidenz. Nervenarzt 1987; 58: 250-255

12. Lazaridis K. Psychiatrische Erkrankungen bei ausländischen Männern. Mensch, Medizin und Gesellschaft 1988; 13

13. Özek M. Ein kasuistischer Beitrag zum Problem psychiatrischer Auffälligkeiten der türkischen Arbeitnehmer in der BRD. In: Eris A, Gökelma Y (Hrsg.). Migranten und Gesundheit. Bremen, Merhaba, 1988: 71-79

14. Slutzki CE. Psychologische Phasen der Migration und ihre Auswirkungen. In: Hegemann T, Salman R (Hrsg.). Transkulturelle Psychiatrie. Bonn, Psychiatrie-Verlag, 2001: 101-115

\section{Korrespondenzadresse:}

PD Dr. Christian Haasen

Klinik für Psychiatrie \& Psychotherapie

Universitäts-Klinikum Hamburg-Eppendorf

Martinistr. 52

20246 Hamburg

haasen@uke.uni-hamburg.de
Georg Thieme Verlag KG

Rüdigerstraße 14

70469 Stuttgart

Telefon (0711) 89 31-0

Telefax (0711) 8931-322

Verantwortliche Schriftleitung:

Markus Gastpar, Essen

ürgen Fritze, Pulheim

Erweiterte Schriftleitung:

Günther Faust, Mainz

Manfred Fichter, Prien am Chiemsee

Andreas Hufnagel, Essen

Joachim Morgner, Dresden

Götz-Erik Trott, Aschaffenburg

\section{Wissenschaftlicher Beirat:}

Thomas Becker, Günzburg; Horst Berzewski, Berlin; Lothar Blaha, Deggendorf; Hans-Dieter Brenner, Bern; Pas quale Calabrese, Bochum; Klemens Dieckhöfer, Bonn; Tilman Elliger, Köln; Volker Faust, Ravensburg; Franz Joseph Freisleder, München; Michael Geyer, Leipzig; Ralf Gold, Göttingen; Iver Hand Hamburg; Kurt Heinrich, Düsseldorf; Paul Hoff, Zürich; Wolfgang Jost, Wiesbaden; Siegfried Kaumeier, Merzig; Gerd Laux, Wasserburg/München; Matthias R. Lemke, Bonn; Walter E. Müller, Frankfurt am Main; Thomas Müller, Bochum;

Dieter Naber, Hamburg; Hartmut Radebold, Kassel; Helmut Remschmidt, Marbold, Kassel; Helmut Remschmidt, Mar-
burg; Peter Riederer, Würzburg; Eckart burg; Peter Riederer, Würzburg; Eckart
Rüther, Göttingen; Jörn Peter Sieb, StralRüther, Göttingen; ]örn Peter Sieb, Stral-
sund; Wolfgang Weig, Osnabrück; Wolfsund; Wolfgang Weig, Osnabrück; Wolf-
gang Werner, Merzig; Roland Wörz, Bad Schönborn; Manfred Wolfersdorf, Bayreuth

Bestellungen: Über den Buchhandel oder direkt beim Verlag

Bezugsbedingungen:

Einzelheft $€ 10,-+$ Porto.

lahresabonnement $€ 72$

inkl. MwSt

inkl. MwSt. und Porto.

gegen Nachweis und Bankeinzug

€ 47,- inkl. MwSt. und Porto.

Ausland zuzüglich Versandkosten

(cash with order) $€ 18,80$ (Europa)

bzw. $€ 49,80$ (Airlift).

Der Abonnementpreis umfasst $12 \mathrm{Ka}$ lendermonate (Mindestlaufzeit). Abonnements laufen weiter, wenn nicht 3 Monate vor lahresende eine Abbestellung beim Verlag vorliegt.
lute

Bei Nichtbelieferung im Falle höherer Gewalt, bei Störungen des Betriebsfriedens, Arbeitskampf (Streik, Aussperrung) bestehen keine Ansprüche gegen den Verlag.

\section{Verlag und Copyright:}

(c) 2004 by Georg Thieme Verlag.

Rüdigerstraße 14, 70469 Stuttgart.

Mit dem Abdruck des Beitrages erwirbt der Verlag das alleinige und ausschließliche Recht für die Veröffentlichung in sämtlichen Publikumsmedien sowie Übersetzungen in fremde Sprachen. Nachdruck, fotomechanische Wiedergabe und Speicherung in den Datenverarbeitungsanlagen, auch auszugsweise, nur nach schriftlicher Genehmigung des Verlages. Die Abbildungen in den Beiträgen stammen, wenn nicht anders vermerkt, von den jeweiligen Autoren. Für Inhalt, Vorschau und Titelseite werden Abbildungen aus den beschriebenen ArAbbildungen aus den beschriebenen Arbeiten verwendet, anderenfalls ist die
Herkunft ebenfalls im Inhaltsverzeichnis deklariert.

\section{Hinweis für Autoren:}

Zur Veröffentlichung in der Zeitschrift angenommen werden unveröffentlichte Originalarbeiten, Übersichtsarbeiten und Kasuistiken, die nicht auch gleichzeitig an anderer Stelle zur Veröffentlichung eingereicht sein dürfen. Richtlinien zur Abfassung des Manuskriptes können bei der Redaktion angefordert werden Die eingereichten Arbeiten für Editorial, Schwerpunkt/Brennpunkt und Übersicht/Originalarbeit werden einem peer review der Schriftleitung unterzogen. Der Inhalt der übrigen Rubriken und der Supplements liegt in der Verantwortung der Redaktion.
Redaktionsleitung:

Günther Buck

Telefon (0711) 89 31-440

Chefredaktion:

Katrin Wolf

Telefon (0711) 89 31-179

Katrin.Wolf@thieme.de

Redaktionsassistenz

Sabine Bischoff

Telefon (0711) 8931-551

Telefax (0711) 8931-322

Internet-Adresse:

http://www.psychoneuro.info

Verantwortlich für den Anzeigenteil:

pharmedia, Anzeigen-

und Verlagsservice $\mathrm{GmbH}$

Anzeigenleitung:

Greta Weller

Telefon (0711) 8931-304

Greta.Weller@pharmedia.de

Zur Zeit gilt Anzeigenpreisliste

1.10 .2004

Zeitschriftenvertrieb:

Telefon (0711) 89 31-321

Herstellung:

Werner Schulz

Telefon (0711) 89 31-331

Satz, Grafik, Layout:

Wolfgang Eckl, Werner Schulz,

Karl-Heinz Zobel

Druck und Verarbeitung:

W. Kohlhammer Druckerei GmbH + Co. 70329 Stuttgart

Bankverbindung:

Deutsche Bank Stuttgart

Konto-Nr. 1420 017, BLZ 60070070

Landesbank Baden-Württemberg

Konto-Nr. 2055 723, BLZ 60050101

Postgiro Stuttgart

Konto-Nr. 45 000-705, BLZ 60010070

Erscheinungsweise: monatlich

Regularly listed in EMBASE

Hinweis:

Wie jede Wissenschaft ist die Medizin ständigen Entwicklungen unterworfen. Forschung und klinische Erfahrung erweitern unsere Erkenntnis, insbesondere was Behandlung, medikamentöse Therapie sowie Diagnostik (Laborwerte etc.) anbelangt. Soweit in dieser Zeitschrift Dosierungen, Applikationen oder Laborwerte erwähnt werden, darf der Leser zwar darauf vertrauen, dass Autoren, Herausgeber und Verlag große Sorgfalt darauf verwandt haben, dass diese Anan Fei Fertigstellung entsprechen. Für Angaben uber Dosierungsanweisungen, Ap plikationsformen und Laborwerte kann vom Verlag jedoch keine Gewähr übernommen werden. Jeder Benutzer ist angehalten, durch sorgfältige Prüfung der Beipackzettel der verwendeten Präparate und ggf. nach Konsultation eines Spezialisten festzustellen, ob die dort gegebene Empfehlung für Dosierungen oder die Beachtung von Kontraindikatiooder die Beachtung von Kontraindikationen gegenüber der Angabe in diese ung ist besonders wichtig bei selten verwendeten Präparaten oder solchen, die neu auf den Markt gebracht worden sind. Jede Dosierung oder Applikation erfolgt auf eigene Gefahr des Benutzers. Laborwerte müssen immer auf Ihre Plausibilität geprüft werden und sind abhängig vom jeweiligen Testgerät bzw. Testkit. Autoren und Verlag appellieren an jeden Benutzer, ihm auffallende Ungenauigkeiten dem Verlag mitzuteilen. 